\title{
A robotics systems design need: a design standard to provide the systems focus that is required for longterm exploration efforts.
}

\author{
H. Charles Dischinger, Jr. \\ Jeffrey B. Mullins \\ George C. Marshall Space Flight Center
}

\begin{abstract}
The United States is entering a new period of human exploration of the inner Solar System, and robotic human helpers will be partners in that effort. In order to support integration of these new worker robots into existing and new human systems, a new design standard should be developed, to be called the Robot-Systems Integration Standard (RSIS). It will address the requirements for and constraints upon robotic collaborators with humans. These workers are subject to the same functional constraints as humans of work, reach, and visibility/situational awareness envelopes, and they will deal with the same maintenance and communication interfaces. Thus, the RSIS will be created by discipline experts with the same sort of perspective on these and other interface concerns as human engineers.
\end{abstract}

\section{INTRODUCTION}

Is has been thirty-two years since humans last walked on another planetary surface or even left Low Earth Orbit. That last effort was intentionally short-duration; the Apollo mission goals did not include permanent human presence in space. In the intervening years, Europeans, Americans, and Russians have tested and demonstrated a technical capacity to perform short and long-term operations in space; the International Space Station represents a continuation of the Soviet intention to establish a permanent presence. At the same time, robotic exploration of the Solar System has continued to characterize the environments humans will eventually enter. For all of this time, there has been a constituency in all the spacefaring nations for long-duration human presence beyond Low Earth Orbit. There has been little support for this effort among the space agencies, however. Certainly part of the reason for this has been the fear on the part of these agencies of program cancellation. There has also been a sense that an "Apollo-type" program lacks staying-power, by its very nature, and that sense has caused temerity about repeating these types of human missions, which might result in withdrawing from the field for a very long time.
In January 2004, United States President George Bush proposed an incremental approach to exploration that, if properly implemented, would develop the infrastructure for exploration as steps are made and provide opportunities for staying, rather than withdrawing. This effort will take advantage of technologies that have been developed for terrestrial purposes. Among these is the use of worker robots that will be assistants to and caretakers for human space explorers. These robots will need to fit into a vast system architecture that includes habitats, human work areas, and exploration vehicles. They will need to be able to interface with these systems appropriately, in order to perform their desired functions. The most logical way to assure the robot designs conform to the mission, and hence system, requirements is to develop a design standard for robots.

\section{RATIONALE}

PRECEDENCE OF STANDARDS FOR HUMANCENTERED DESIGN - Humans are extremely complex, but this has not prevented the development of standards for the design of systems with which we interact. In the case of design of spacecraft, the need for such standards was recognized as early as 1966, when NASA published MSFC-STD-267A'. This and like documents eventually evolved into the Man-Systems Integration Standards (MSIS), NASA-STD- $3000^{2}$. This document is an Agency-wide standard and thus applies to all NASA human spaceflight programs. That is, the requirements for new programs, such the International Space Station, are derived directly ("flowed-down") from this document. It covers development of all hardware that humans in NASA vehicles interact with in Low Earth Orbit, from the space vehicles themselves to the payloads that astronauts manipulate to tools required for the deployment and maintenance of these systems. Specifically, it defines the bounds for workstation layout, communication methodologies, and life support, for both intravehicular and extravehicular activities. In support of these requirements, it fully documents human capabilities in the space environment, including anthropometry, flexibility and reach envelopes, resource requirements, temperature tolerance, and radiation susceptibility. This background is important for the establishment of the design requirements, and it is often 
essential to their interpretation. The creation of these standards very likely seemed unnecessary at the time they were developed. Even today, they are seen as statements of "common sense" (a capability the authors find startlingly uncommon) for which no requirement is necessary, and the claim is often made that they are constrictive of design creativity. Nevertheless, their contributions to mission success and prevention of loss of life are well established.

ANALOGY BETWEEN ROBOTICS SYSTEMS AND HUMAN SYSTEMS - Collaborative, or helper, worker robots will perform many of the same functions as humans, except that they will not often work completely autonomously, at least in the nominal mission. In this sense, they will act as assistants to the human, as an apprentice might. Some will carry tools and other supplies and provide the proper one when called for. They will display procedures and other technical information for humans performing maintenance tasks or conducting science. On planetary surfaces, some will serve as carts for the human and for the units being maintained; that is, they will transport spare parts to be changed out and return the worn-out or damaged parts to a depot. During maintenance missions, they will team with humans, dividing tasks with them, once a certain level of robotic sophistication is reached. Some will serve as search-and-rescue and medical technicians for humans and for other robots. Some highly specialized robots will serve as medical assistants for complex procedures, being teleoperated by physicians remote to the exploration system ${ }^{3}$.

These worker robots will be subject to the same functional constraints as humans of work, reach, and visibility/situational awareness envelopes. That is, they must be able to fit into a work area, reach the worksite to be actuated, and perceive both the location of the worksite and its qualities: bolt head size and condition, lever position, whether a cover is present, and so forth. They will deal with the same maintenance and communication interfaces as humans. That is, they will demate and mate electrical, data, and fluid connectors. They will actuate bolts and other fasteners. They will remove and replace components. Finally, they must obtain procedures for these and other tasks from external sources and store the procedures in memory. Each of these tasks is, of course, an analogue to human tasks, and each task implies a set of requirements. The similarity to the MSIS should not be missed; the design requirements associated with human task performance were derived from scenarios of the tasks that humans might be expected to perform in Low Earth Orbit. An understanding of the function of and tasks to be performed by helper robots will drive out the requirements those tasks imply. This definition of the tasks, in the form of a Critical Task Analysis, is a typical responsibility of the Human Engineer during the requirements definition phase of a design project ${ }^{4}$. This process ordered and reduced the scope of the otherwise seemingly boundless job of developing requirements for human design, and the same methodology should be employed for requirements relating to robots.

PREVIOUS ROBOTICS STANDARDS - Early in the International Space Station program, a robotics standard was developed and titled the Robotics Systems Integration Standard ${ }^{5}$. This document described only standards for robots associated with the Station program and was thus limited in scope. In NASA parlance, it was a "Level II" document: one associated with a single program within a single agency. While the document began to address many of the issues that must be considered for a general robotics standard, it did so only within the context of the extant Space Station. The proposed standard would be superior to the program and could even be superior to the agency. It could be developed by and agreed to by the various partners collaborating in the exploration effort. Whether done at the agency level or at the international partner level, it naturally will be much more general and broad-ranging than the International Space Station standard.

OTHER EFFORTS - Introductory texts in robotics (e.g., $6,7)$ address primarily the theory of design of a single robot. Discussion of integration of several to many robots into a system is limited to the development and operation of production lines. This is consistent with the current state of capabilities of robots and of designers to develop them. However, the types of interactions that the proposed new standard would address are beyond the highly repetitive and tightly-constrained tasks of the production line robots, and they imply considerably more collaborative capabilities than are found in existing mobile robots. The standard would address the ways robots interact with each other, with humans, and with other system-level hardware and software (here, "exploration system" refers to uncrewed planetary satellites and propulsion systems, as well as inspace and planetary crewed modules). It is important to emphasize that this standard will require no particular capability of individual robots and that it will not attempt to describe how to build a robot. Rather, it will allow robots to be designed so that they conform to the exploration system in an integrated way. This is important both in the near-term and as advanced capabilities are developed. Thus, there would be no requirement that a robot be developed that is capable of carrying out a complete search-and-rescue mission for a missing and injured human in a completely autonomous manner. It is conceivable that such a worker will become available at some time in the future, but all the capabilities implicit in this sort of mission scenario will not appear at once. In fact, the first helpers can reasonably be expected to have a level of capability that is only slightly greater than that of the robotic arm on the ISS or of the Spirit rover, each of which requires a human operator. The point of the standards will be to 
assure that the functionalities that exist are properly integrated into the exploration system and that new features can be added seamlessly as they become available.

APPLICABILITY - The proposed document should be known as the Robotics Systems Integration Standards (RSIS), to allow it to be recognized as an agency-level ("Level I") document analogous to the MSIS. It will address worker robots which operate collaboratively with humans, even though they may ultimately possess a great deal of autonomous capability. These helper robots will be those that support human activities, by assisting in and performing maintenance tasks on the exploration system by acting as research assistants, and by serving as life-protection and safety devices for humans. Since this type of robotic assistant will be part of essentially all future space systems, this volume will have far-flung ramifications.

LIMITATIONS - It is recognized that such a standard cannot apply to all conceivable robots, and design solutions for some robots would be constrained if the standard were made applicable to all robots. The idea is that robots that fit the "worker" category should be subject to the standards. The criterion is whether humans or exploration system resources need to interface directly with the robot. Thus, fully-autonomous explorers, which never encounter humans once released or which do not require maintenance or resources if they do, would not be subject. Likewise, it is easy to imagine robots which are constantly in the human environment but for which the RSIS would be inappropriate. For example, free-living, centimeter-sized cleaning devices or expendable snake robots for exploring small spaces might be useful. Since these do not conform to the "Helper worker model," both of these and similar independent robots would be exempt, or the standard would be applicable only to the extent that they are not independent; e.g., resource-allocation requirements might apply, if these robots depend upon power supply from the exploration system.

Both the MSIS and the RSIS have the goal of assuring a well-integrated system with a high likelihood of mission success, and the development of the two documents can follow similar paths. The MSIS defines the constraints on system design that are imposed by human limitations and capabilities. Robots do not have the same limitations, but they do have limitations. A major undertaking of the development of this document must be the characterization of these constraints. The human constraints and capabilities described in MSIS constitute what designers often call "common sense," but a cursory reading of the document reveals many features of human physiology and psychology that are, in fact, not intuitive. Similarly, the definition of the limitations and capacities of robotic helpers will form the assumptions upon which the requirements are based. Since these requirements will have a profound impact on the evolution of helper robots over the course of many years, their development is an important task. Some might argue that freedom to create a variety of systems will result in the best technical solutions, but there is little historical evidence to support this thesis. Many familiar, if simplistic, examples of this can be found in the field of consumer electronics. For example, while Betamax $(\subset$ was widely regarded as the superior commercial video recording technology in the 1970 s, it has essentially disappeared today.

\section{STRUCTURE OF RSIS AND TOPICS TO BE ADDRESSED}

There should be three volumes in the initial release of this document. The first will deal with the design considerations and design requirements for the assembly and integration of worker robots. The second should address the design considerations and design requirements for development of subsystems and components to be manipulated by (maintained, transported, or stowed by) robots. The third will be a set of appendices addressing the sources of the

requirements found in the other two volumes, glossaries, acronym lists, unresolved issues, and the like.

VOLUME 1 - The first volume will describe a standardized set of robotic functions, capabilities, and constraints. It will relate these to mission concepts and to interfaces with other hardware and software, in order to define the requirements for robot design that support the mission goals. Sections of the volume will address propulsion, guidance and navigation, command and control, communications, mechanisms, end effectors, and sensors. There should be at least one section each that addresses interfaces with humans, other worker robots, and exploration system hardware and software. Note that the section on human:robot interfaces is critical. It is expected that, in addition to performing other tasks, some robots will transport or be transported by humans, some will provide them temporary life support, some will perform surgery, some will act as task assistants, and most will understand and react to human commands (verbal, as well as digital) and provide information to humans in understandable formats. These aspects of the standards will need to be addressed in human/computer interface standards of the MSIS, as well as in this volume.

In addition, there will be at least one section on requirements for maintenance of the robots by humans (and, ultimately, other robots), but this will only address those requirements that are unique to robot maintenance and are not found in the MSIS. It should be noted, however, that the MSIS may need to be revised. One goal for maintainability of all hardware, robot and exploration system, will be that tool interfaces will be consistent across maintenance workers. That is, 
humans and worker robots should expect to encounter exactly the same tool interfaces when they approach a worksite, whether it be a power supply subsystem site, a robot rover cart, or a habitat environmental control subsystem. Limitation of the number of tools required to perform tasks is a primary goal of the development of this standard, so there will be a section on tool definition. This is important for several reasons: tools, while small compared to modules, are mass that must be lifted from the earth for the foreseeable future and are therefore costly; tools must be transported to a worksite, and the capacity of a toolbox, even on a rover robot, is limited; and keeping the number of tools needed for a task small reduces the likelihood that workers will arrive at the worksite with the wrong tool! One section will address the levels of autonomy a particular design can achieve. Many robots may be assemblable from a standard design to meet the mission goals. That is, the various subsystems for classes of robots should be available for a mission designer basically off-the-shelf. This sort of standardization will reap huge benefits for long-duration missions in logistics and spares, maintenance, and ground and flight crew training. While this approach can result in a wide variety of individual robots, the assembly from off-the-shelf components makes them more affordable and more reliable. The flow-down benefits to short-term missions and the design of free-existing robots are apparent but are not drivers.

There may need to be special sections of Volume I to address special topics. For example, search-and-rescue robots may form a special class and need to be addressed separately. Also, other scenario-derived requirements may surface, such as the need for some workers to have transponder capability, to allow humans and other robotic workers to work outside line-of-site of the base station.

VOLUME II - Design requirements for developers of exploration systems that will be serviced by robots will be covered in the second volume, This volume is, for all practical purposes, analogous to NASA-STD-3000 in its impact on design. All designers of subsystems with which robots will interface will need to conform to this volume. It will address the work envelopes that the standard robots must perform within, the strength capabilities of these robots, the interfaces they are capable of dealing with on the hardware to be manipulated (fluid, electrical, and data connectors; fasteners; volumes and dimensions; et $c$.), as well as the structural and thermal characteristics of the hardware. These are in almost every way analogous to the design issues addressed in NASA-STD-3000. This is because humans and robots face the same sorts of constraints when performing tasks; both must be able to detect ("see," in the case of humans) and reach a hardware interface to manipulate it, and the manipulable component must conform to the constraints of the human (e.g., hand size, strength) or robot (e.g., tool fitting size, cybernetics).

VOLUME III - This book will contain ancillary data, analogous to that in MSIS Vol. II. This is the background information that documents how requirements in the other volumes were derived. It will thus contain an operations concept section, which includes various robot mission scenarios which form the basis of the requirements. This section is critical to the development process. It is analogous to the task analysis development of the Human Engineer ${ }^{4}$ and the scenariobased requirements development of the systems engineer ${ }^{9}$. It will also list all reference information and the specific requirements they apply to. Another section will provide notes documenting the thought processes of the requirements development team; that is, it will document, in everyday language, what the team thought the requirement meant at the time of development. This volume will serve as a reference for developers of robots and other systems, who must interpret the design requirements in the context of their own mission requirements. Glossaries, acronym lists, open issues, and other relevant information should also be put into Volume III.

\section{SYSTEM COMPLEXITY AND RISKS}

All human exploration involves risk, and complex systems are inherently risky. Failures in the NASA Space Shuttle Programs provide tragic demonstrations of the effects of the combination of these sources of risk. Many other failures of complex systems can be cited; among the most disastrous are the control room failure at the Three Mile Island nuclear power facility and the shooting down of the Iran Air passenger liner by the USS Vincennes. Both accidents occurred because of poor system design, particularly design of the human:machine interface ${ }^{8}$. When it is first developed, any conceivable exploration system will be arguably the most complex system yet created by humans, as ISS is today. Robots will not only add to that system complexity, they will be among the most complex individual components of it. The exploration effort will face a myriad of risks derived simply from the novel environments entered. It can ill afford complexity-driven risks. These cannot be completely avoided, but they can be managed. An axiom of systems engineering is that one of the most effective ways to mitigate the risks in complex systems is through thorough process-driven requirements development early in the program. This is the primary purpose of the RSIS. It should be developed early in the design phase of the exploration program and then levied on all exploration systems. The failure to create such a standard early in an exploration program will at the least result in the very high operations costs associated with a poorly-integrated system. A much more serious consequence would be the loss of life early in the program. Such a disaster will always be tragic, but it 
could also result in public castigation of the agency and possible program abandonment. If this were to be attributable to design integration failures, it would be not only tragic but unconscionable.

\section{DEVELOPMENT OF THE DOCUMENT}

Development of requirements for any project, large or small, demands an understanding of the mission; the basic question is, "what do we want this system to do?" A Concept of Operations document is a useful way to establish mission goals. It is recommended that this be the first step, and that development of subsystem (in this case, robotic) mission scenarios be based on this Concept of Operations. The scenarios will eventually take the form of Critical Task Analyses ${ }^{4}$ for the robots involved in a particular mission. Here, the word "mission" is used to indicate a type of short-term activity of a particular robot or class of robots. Thus a mission might be the collection of subsurface material from the centers of all lunar craters within eighty kilometers of a lunar base and the return of the material. This mission would not be accomplished in a single sortie, and it would be established that the robot(s) will be working collaboratively with humans. This sort of scenario definition will both establish a set of basic requirements (e.g., power needs, lower limits for load-carrying capacities, thermal environments, communication interfaces, et $c$.) and raise a set of questions that need to be further examined through trade studies (will the robot provide human transportation? will it provide human life support?). Note that this activity brings to light requirements on both side of the interface between the robot and its environment. Thus, if it is decided that the robot will provide life support to the human during the translocation phase of the mission, the requirements on the robot will be to provide power, gases, and fluids to the human's suit. It also requires that the human's suit be designed to connect appropriately to the robot. Furthermore, the robot will need to be able to connect to the necessary depot interfaces at the station in order to recharge with consumables, and the depot will be required to provide said interfaces.

This process will be iterative and will be followed for each scenario. The requirements that flow out of them would then be sorted by class and organized into sections within Volumes I \& II of the RSIS. The process itself will be captured in Volume III.

It should be apparent that the development of the RSIS is not a robotics-specialist task. While such subjectmatter experts should be team members, the majority of the effort should fall on systems engineers with a background in human engineering. The issues dealt with by human system designers are exactly those faced by the systems of robots and the environments with which they will interact. Possibly more importantly, the design of human interface with these complex machines is likely the most critical part of the work.

\section{CONCLUSION}

In the next phase of exploration robots will be collaborative assistants to humans. These worker robots will be part of the most complex systems ever created by humans. Failure to properly integrate them into the exploratory systems will not simply be costly; it will likely lead to system failures that could result in mission failure, including loss of human life. A systems engineering perspective will support management of this risk. Specifically, the development of a Robotics Systems Integration Standard will support the proper management of the complexity in these exploration systems.

\section{REFERENCES}

1. 1966. Anon. Standard Human Engineering Design Criteria: MSFC-STD-267A. Marshall Space Flight Center, Alabama.

2. 1987. Anon. Man-Systems Integration Standards, NASA-STD-3000.

3. E.g.,see:

http://www.texastechnology.com/Curriculum/Emergi ng Technology/Robotics Education/robotatwork/ind ex.asp?pageid=medrobotext.htm and http://ranier.hq.nasa.gov/Telerobotics page/Technol ogies/0524.html

4. 1999. Anon. Human Engineering Program Process and Procedures, MIL-HDBK-46855A.

5. 1998. Muchinsky, M. M., ed. Space Station Robotic Systems Integration Standard. SSP 30550.

6. 1996. McKerrow, P.J. Introduction to Robotics. Addison-Wesley Publishing Co., Sydney.

7. 2000. Nehmzow, U. Mobile Robotics: a Practical Approach. Springer-Verlag, London.

8. 2002. Mulgaonkar, P. and H. Dobbs, eds. Ad hoc Study on Human Robot Interface Issues. Army Science Board, Arlington, Va.

9. 1995. Carroll, J.M., ed. Scenario-based Design. John Wiley \& Sons, Inc. New York.

\section{CONTACT}

H. Charles Dischinger, Jr.: NASA/Marshall Space Flight Center, EV11, MSFC, AL 35812. charles.dischinger@nasa.gov 